\title{
Kohn-Sham potentials in exact density-functional theory at non-integer electron numbers
}

\author{
Tim Gould \\ Qld Micro- and Nanotechnology Centre, Griffith University, Nathan, Qld 4111, Australia* \\ Julien Toulouse \\ Sorbonne Universités, UPMC Univ Paris 06, UMR 7616, \\ Laboratoire de Chimie Théorique, F-75005 Paris, France \\ CNRS, UMR 7616, Laboratoire de Chimie Théorique, F-75005 Paris, France
}

\begin{abstract}
Within exact electron density-functional theory, we investigate Kohn-Sham (KS) potentials, orbital energies, and non-interacting kinetic energies of the fractional ions of $\mathrm{Li}, \mathrm{C}$ and $\mathrm{F}$. We use quantum Monte Carlo densities as input, which are then fitted, interpolated at non-integer electron numbers $N$, and inverted to produce accurate KS potentials $v_{\mathrm{s}}^{N}(\boldsymbol{r})$. We study the dependence of the KS potential on $N$, and in particular we numerically reproduce the theoretically predicted spatially constant discontinuity of $v_{\mathrm{s}}^{N}(\boldsymbol{r})$ as $N$ passes through an integer. We further show that, for all the cases considered, the inner orbital energies and the non-interacting kinetic energy are nearly piecewise linear functions of $N$. This leads us to propose a simple approximation of the KS potential $v_{\mathrm{s}}^{N}(\boldsymbol{r})$ at any fractional electron number $N$ which uses only quantities of the systems with the adjacent integer electron numbers.
\end{abstract}

PACS numbers: 31.15.ae,31.15.E-,31.15.ve

Over the past few decades, Kohn-Sham (KS) [1] density-functional theory (DFT) [2] has become one of the most important tools in electronic-structure theory. Given the overwhelming popularity of density-functional approximations (DFAs) (e.g., PBE [3], hybrids [4]), surprisingly few studies have been dedicated to the detailed properties of the exact KS system. This is despite the fact that unusual properties [5] of the fictious noninteracting $\mathrm{KS}$ system serve a vitally important role in reproducing the quantum mechanical properties of the interacting system in cases where degeneracies are present in the ground state, or where electrons are added and removed. In this paper we study exact KS DFT properties of difficult, open quantum systems with degenerate ground states - specifically open-shell atoms with noninteger electron numbers. A primary aim is to provide guidance for the construction of new DFAs.

In quantum mechanics, open electronic systems with a non-integer average number of electrons naturally arise, for example, as fragments from a molecular dissociation in entangled quantum states. In particular, in DFT the study of systems with fractional electron numbers is of great importance for a better understanding of the theory (for a recent review, see Ref. 6). For such fractional systems, Perdew et al. [7] proved[8] that the energy is a piecewise linear function of the electron number between the adjacent integers. This lead to the theoretical prediction of the discontinuity of the KS potential as the electron number passes through an integer, with many important physical consequences concerning the description of the fundamental gap [9, 10], molecular dissociation [7] or charge-transfer excitations [11]. This also lead

\footnotetext{
* t.gould@griffith.edu.au
}

to the explanation that the underestimation of energies obtained with the usual semilocal DFAs for delocalised densities is a consequence of their deviations from the exact piecewise linear behavior of the energy [12-16]. These understandings have guided the design of improved DFT approximations [17-22].

Although the piecewise linear energy curve and the discontinuity of the KS potential have been widely discussed in the literature, little else is known about the form of the exact KS potential as a function of the fractional electron number. In this paper, we fill this gap by providing and analysing accurate KS potentials as a function of the electron number for the fractional ions of $\mathrm{Li}, \mathrm{C}$ and $\mathrm{F}$, exploring a range from 2 to 10 electrons. For all these systems, we numerically reproduce the discontinuity of the KS potential. We then propose a simple approximation of the KS potential at any fractional electron number which uses only quantities of the systems with the adjacent integer electron numbers.

KS DFT for fractional electron numbers. The groundstate energy of a system with a fractional number of electrons $N=M+f$ (where $M$ is an integer and $0 \leq f \leq 1$ ) can be defined in the zero-temperature grand-canonical ensemble formalism as [7, 22, 23] (see also Refs. 24 and 25 for an alternative view)

$$
E^{N}=\min _{\hat{\Gamma} \rightarrow N} \operatorname{Tr}\left[\hat{\Gamma}\left(\hat{T}+\hat{V}_{\mathrm{ext}}+\hat{W}_{\mathrm{ee}}\right)\right]
$$

where Tr denotes the trace and the search is over all normalized ensemble fermionic density matrices $\hat{\Gamma}$ yielding $N$ electrons, i.e. $\operatorname{Tr}[\hat{\Gamma} \hat{N}]=N$ where $\hat{N}$ is the number operator. In Eq. (1), $\hat{T}$ is the kinetic energy operator, $\hat{V}_{\text {ext }}$ is the external potential operator, and $\hat{W}_{\text {ee }}$ is the electron-electron interaction operator. With the usual 
assumption that the ground-state energy for integer electron numbers in a fixed external potential is a convex function, $E^{M} \leq\left(E^{M+1}+E^{M-1}\right) / 2$, the minimizing density matrix is obtained as a linear interpolation between ground-state density matrices of the $M$ and $(M+1)$ electron systems

$$
\hat{\Gamma}^{N}=(1-f) \hat{\Gamma}^{M}+f \hat{\Gamma}^{M+1},
$$

where $\hat{\Gamma}^{M}=\sum_{e} w_{e}\left|\Psi_{e}^{M}\right\rangle\left\langle\Psi_{e}^{M}\right|$ (with $\left.\sum_{e} w_{e}=1\right)$ is made of the possibly degenerate $M$-electron ground states, and similarly for $\hat{\Gamma}^{M+1}$. Equation (2) immediately implies that the ground-state energy and the one-electron density are also piecewise linear in $f$

$$
\begin{aligned}
E^{N} & =(1-f) E^{M}+f E^{M+1}, \\
n^{N}(\boldsymbol{r}) & =(1-f) n^{M}(\boldsymbol{r})+f n^{M+1}(\boldsymbol{r}),
\end{aligned}
$$

where $n^{M}(\boldsymbol{r})$ is the density corresponding to the density matrix $\hat{\Gamma}^{M}$, and similarly for $n^{M+1}(\boldsymbol{r})$. The freedom in the choice of the ensemble weights $w_{e}$ can be used to impose symmetries. For example, for atoms with an incomplete p shell, one can choose $w_{e}$ so as to obtain a spherically symmetric density $n^{M}(r)$.

In KS DFT, an universal functional can be defined for any fractional electron density $n(\boldsymbol{r})$ with the constrainedsearch formalism [7]

$$
F[n]=\min _{\hat{\Gamma} \rightarrow n} \operatorname{Tr}\left[\hat{\Gamma}\left(\hat{T}+\hat{W}_{\mathrm{ee}}\right)\right],
$$

where $\hat{\Gamma} \rightarrow n$ refers to all normalized ensemble fermionic density matrices $\hat{\Gamma}$ yielding the density $n(\boldsymbol{r})$, i.e. $\operatorname{Tr}[\hat{\Gamma} \hat{n}(\boldsymbol{r})]=n(\boldsymbol{r})$ where $\hat{n}(\boldsymbol{r})$ is the density operator. Decomposing this functional as

$$
F[n]=T_{\mathrm{s}}[n]+E_{\mathrm{Hxc}}[n],
$$

where $T_{\mathrm{s}}[n]=\min _{\hat{\Gamma} \rightarrow n} \operatorname{Tr}[\hat{\Gamma} \hat{T}]$ is the KS non-interacting kinetic-energy functional and $E_{\mathrm{Hxc}}[n]$ is the remaining Hartree-exchange-correlation functional, leads to the KS expression for the exact ground-state energy

$$
E^{N}=\min _{\hat{\Gamma}}\left\{\operatorname{Tr}\left[\hat{\Gamma}\left(\hat{T}+\hat{V}_{\mathrm{ext}}\right)\right]+E_{\mathrm{Hxc}}\left[n_{\hat{\Gamma}}\right]\right\}
$$

where the functional $E_{\mathrm{Hxc}}$ is evaluated at the density coming from $\hat{\Gamma}$. The minimizing density matrix is assumed to have the form

$$
\hat{\Gamma}_{\mathrm{s}}^{N}=(1-f) \hat{\Gamma}_{\mathrm{s}}^{M, f}+f \hat{\Gamma}_{\mathrm{s}}^{M+1, f},
$$

where $\hat{\Gamma}_{\mathrm{s}}^{M, f}=\sum_{e} w_{e}\left|\Phi_{e}^{M, f}\right\rangle\left\langle\Phi_{e}^{M, f}\right|$ is a density matrix made of $M$-electron single-determinant wave functions (which depend on $f$ ), and similarly for $\hat{\Gamma}_{\mathrm{s}}^{M+1, f}$, and again the ensemble weights $w_{e}$ can be used to impose symmetries. All the $M$ - and $(M+1)$-electron single determinants are constructed from a common set of KS orbitals $\left\{\phi_{i}^{N}(\boldsymbol{r})\right\}$ determined by (in atomic units)

$$
\left[-\frac{1}{2} \nabla^{2}+v_{\mathrm{s}}^{N}(\boldsymbol{r})\right] \phi_{i}^{N}(\boldsymbol{r})=\varepsilon_{i}^{N} \phi_{i}^{N}(\boldsymbol{r}),
$$

where $v_{\mathrm{s}}^{N}(\boldsymbol{r})$ is the KS potential,

$$
v_{\mathrm{s}}^{N}(\boldsymbol{r})=v_{\mathrm{ext}}(\boldsymbol{r})+\frac{\delta E_{\mathrm{Hxc}}\left[n^{N}\right]}{\delta n(\boldsymbol{r})},
$$

and $\varepsilon_{i}^{N}$ are the KS orbital energies $\left(\varepsilon_{i}^{N} \leq \varepsilon_{j}^{N}\right.$ for $\left.i<j\right)$. In Eq. (10), the KS potential is evaluated at the exact density $n^{N}(\boldsymbol{r})$ which is also the density given by the KS density matrix $\hat{\Gamma}_{\mathrm{s}}^{N}$ and can be written in terms of the KS orbitals and fractional occupation numbers $\left\{f_{i}\right\}$

$$
n^{N}(\boldsymbol{r})=\sum_{i} f_{i}\left|\phi_{i}^{N}(\boldsymbol{r})\right|^{2}
$$

where $f_{i}=2$ for the $N_{\text {inner }}$ lowest inner doubly occupied orbitals, $\sum_{h} f_{h}=N-2 N_{\text {inner }}$ for the fractionally occupied degenerate highest occupied orbitals (HOMOs), and $f_{i}=0$ for the remaining unoccupied orbitals. Unlike other work [26, 27], we use a spin-restricted open-shell formalism, and thus avoid dealing with spin dependence.

In principle, if density variations changing the electron number are allowed in the functional derivative of Eq. (10), the KS potential $v_{\mathrm{s}}^{N}(\boldsymbol{r})$ including its spatialconstant component is uniquely determined. In practice, for any fixed $N$, if an accurate electron density $n^{N}(\boldsymbol{r})$ is known, one can find the corresponding $\mathrm{KS}$ potential $v_{\mathrm{s}}^{N}(\boldsymbol{r})$ obeying $v_{\mathrm{s}}^{N}(|\boldsymbol{r}| \rightarrow \infty)=0$ by ensuring that the orbitals satisfying Eq. (9) give the density through Eq. (11). In this work, we make use of this mapping to obtain accurate KS potentials $v_{\mathrm{s}}^{N}(\boldsymbol{r})$, orbital energies $\varepsilon_{i}^{N}$, and KS kinetic energies $T_{s}^{N} \equiv T_{\mathrm{s}}\left[n^{N}\right]$ as a function of the fractional electron number $N$.

Computational method. We consider atoms with external potential $v_{\text {ext }}(r)=-Z / r$. Using Eq. (4), the density for an ion with any fractional electron number between $Z-1$ and $Z+1$ can be obtained from the densities for $Z-1, Z$, and $Z+1$ electrons. We thus first calculate (spherically averaged) accurate densities $n^{M}(r)$ for these integer electron numbers using quantum Monte Carlo (QMC). Specifically, the densities are calculated in diffusion Monte Carlo with an improved statistical estimator [28], using Jastrow full-valence complete-activespace wave functions fully optimized at the variational Monte Carlo level [29]. As an accurate inversion requires a density to be free from statistical errors, we fit these QMC densities with a simple, yet asymptotically accurate (as $r \rightarrow \infty$ ) function that ensures that the density corresponds to a HOMO with the appropriate eigenen$\operatorname{ergy} \varepsilon_{h}$, and to a KS potential with correct asymptotic behavior $v_{\mathrm{s}}^{M}(r \rightarrow \infty) \sim(-Z+M-1) / r$. The fitting function and parameters given in the supplementary material [30] give densities that are accurate to within the QMC statistical error.

After obtaining the density at fractional electron numbers $n^{N}(r)$ via Eq. (4), we calculate the KS potential by using a numerically stabilised modification of Wang and Parr's iterative approach [31]. The KS potential at iteration $m+1$ is found 

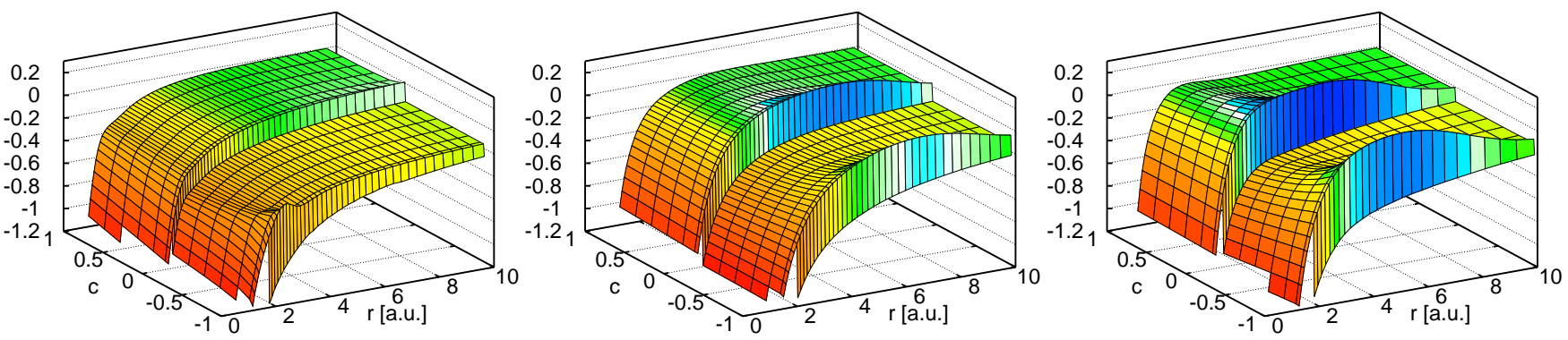

FIG. 1. (Color online) KS potentials $v_{\mathrm{s}}^{Z+c}(r)$ [hartree] as a function of the radial distance $r$ and the fractional excess electron number $c=N-Z$ for Li (left), $\mathrm{C}$ (middle) and $\mathrm{F}$ (right). Colors indicate height (from red to green for $v_{\mathrm{s}}^{Z+c}(r)<0$, white for $v_{\mathrm{s}}^{Z+c}(r) \approx 0$, blue for $\left.v_{\mathrm{s}}^{Z+c}(r)>0\right)$.

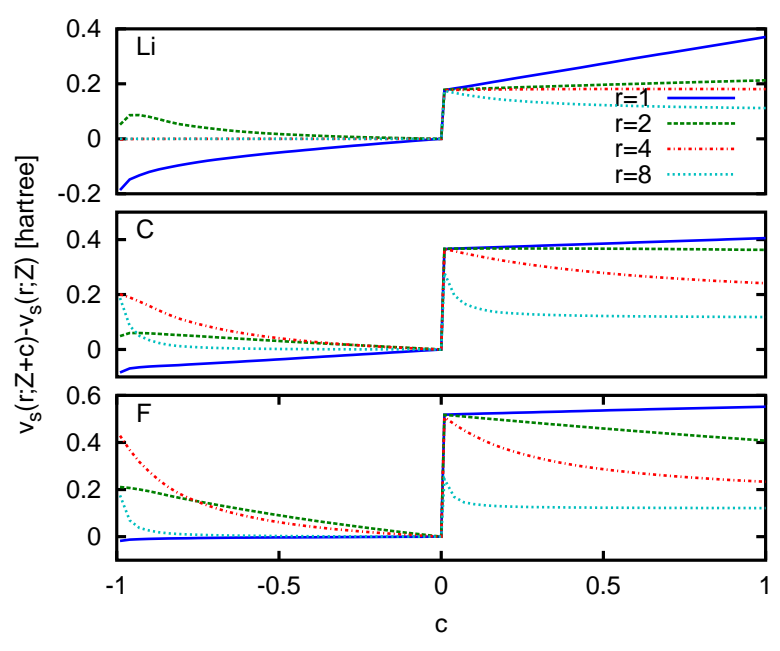

FIG. 2. (Color online) Change in Kohn-Sham potentials $v_{\mathrm{s}}^{Z+c}(r)-v_{\mathrm{s}}^{Z^{-}}(r)$ as a function of $c>-1$ for Li (top), C (middle) and $\mathrm{F}$ (bottom), for selected values of $r$.

from the quantities at the previous iteration $m$ through $v_{\mathrm{s}}^{N, m+1}(r)=v_{\mathrm{s}}^{N, m}(r)+Q \frac{n^{N}(r)-n^{N, m}(r)}{G^{N, m}(r)}$, where $G^{N, m}(r)=\sum_{i} f_{i}\left|\phi_{m}^{N, m}(r)\right|^{2} / \varepsilon_{i}^{N, m}+\max \left[0,\left(1 / \varepsilon_{h}-\right.\right.$ $\left.\left.1 / \varepsilon_{h}^{N, m}\right)\left\{n^{N}(r)-n^{N, m}(r)\right\}\right]$, and $Q>0$ is a convergence parameter. By starting from fractional LEXX [21] potentials and orbitals we achieve $\int \mathrm{d} \boldsymbol{r}\left|n^{N}(\boldsymbol{r})-n^{N, m}(\boldsymbol{r})\right|<$ $10^{-6}$, albeit with increasing errors in $v_{\mathrm{s}}^{N}$ for $N \leq Z-0.8$.

Results and discussion. In Figure 1 we show the KS potentials $v_{\mathrm{s}}^{Z+c}(r)$ (plots of its components are shown in the supplementary material [30]) as a function of the radial distance $r$ and the fractional excess electron number $c=N-Z$ where $-1 \leq c \leq 1$, i.e. for ions from $\mathrm{A}^{+}$through $\mathrm{A}$ to $\mathrm{A}^{-}$ for $\mathrm{Li}, \mathrm{C}$ and $\mathrm{F}$. Calculations are performed for $c \in$ $\{-1,-0.99,-0.95,-0.8,-0.6,-0.4,-0.2,0,0.01,0.05$, $0.2,0.4,0.6,0.8,1\}$.

The most obvious feature of these plots is the presence of a discontinuity in the potentials as the electron number $N$ crosses an integer. Let us concentrate on the discontinuity at $N=Z$, i.e. $c=0$. At first sight, in Figure 1 , this discontinuity seems to be dependent on $r$, decreasing at large distances. To analyse the discontinuity more precisely we plot in Figure 2 the quantity $v_{\mathrm{s}}^{Z+c}(r)-v_{\mathrm{s}}^{Z^{-}}(r)$ as a function of $c$ (interpolated in $-0.99 \leq c \leq 0$ and $0.01 \leq c \leq 1)$ for selected values of $r$. It is now clear that, if we extrapolate the plots from $c>0$ toward the limit $c \rightarrow 0^{+}$, the results are consistent with the KS potential having a constant discontinuity

$$
\Delta^{Z}=v_{\mathrm{s}}^{Z^{+}}(r)-v_{\mathrm{s}}^{Z^{-}}(r)=I-A,
$$

at $N=Z$, independent of $r$. For the open-shell systems considered here, the discontinuity $\Delta^{Z}$ is equal to the difference between the ionization energy $I$ and the electronic affinity $A$ of the neutral atom. Therefore, the present results numerically reproduce the theoretically predicted $[32,33]$ spatially constant discontinuity of the KS potential when the electron number $N$ crosses an integer $M=3,6$, or 9 , which had been so far numerically observed only for $M=1[32-34]$. The values of $\Delta^{Z}$ are found to be $0.178,0.368$ and 0.519 hartree, for $\mathrm{Li}, \mathrm{C}$ and $\mathrm{F}$, respectively, very close to $0.175,0.366$ and 0.516 hartree found using $I-A$.

Beside the discontinuity, it is interesting to note that the potential is sensitive to the order of the limits $c \rightarrow 0^{+}$ and $r \rightarrow \infty$ [33]. It is clear from Figures 1 and 2 that as $r$ increases the KS potentials go to zero in a larger and larger range of $c$, except near the limit $c \rightarrow 0^{+}$where it becomes more and more curved. This is understandable since $v_{\mathrm{s}}^{Z+c}(r)-v_{\mathrm{s}}^{Z^{-}}(r)$ must go to $\Delta^{Z}$ in the limit $c \rightarrow$ $0^{+}$, but at the same time, for any finite value of $c$, the KS potential for $M<N \leq M+1$ must have the same asymptotic behavior as the KS potential for the $(M+1)$ electron system, $v_{\mathrm{s}}^{N}(r \rightarrow \infty) \sim v_{\mathrm{s}}^{(M+1)^{-}}(r) \sim(-Z+$ $M) / r$ [35], and therefore must go to zero for large $r$.

In Figure 3 we plot as a function of $c$ the difference between the orbital energies at the fractional electron number $Z+c$ and their neutral atomic values: $\varepsilon_{i}^{Z+c}-\varepsilon_{i}^{Z^{-}}$. As expected, all orbital energies have a discontinuity of $\Delta^{Z}$ at $c=0$. As required by theory the HOMO energy is constant, within numerical noise, between integer electron numbers: $\varepsilon_{h}^{N}=\varepsilon_{h}^{M^{-}}+\Delta^{M}=\varepsilon_{h}^{(M+1)^{-}}$. Interestingly, we find that the energies of the inner orbitals 


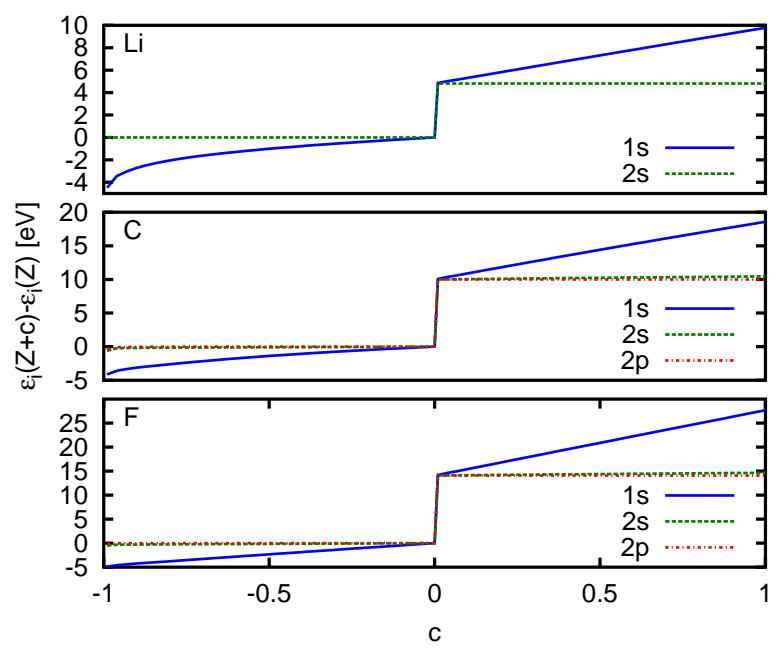

FIG. 3. (Color online) Change in Kohn-Sham orbital energies for all orbitals $\varepsilon_{i}^{Z+c}-\varepsilon_{i}^{Z^{-}}$as a function of $c>-1$ for Li (top), $\mathrm{C}$ (middle) and F (bottom).

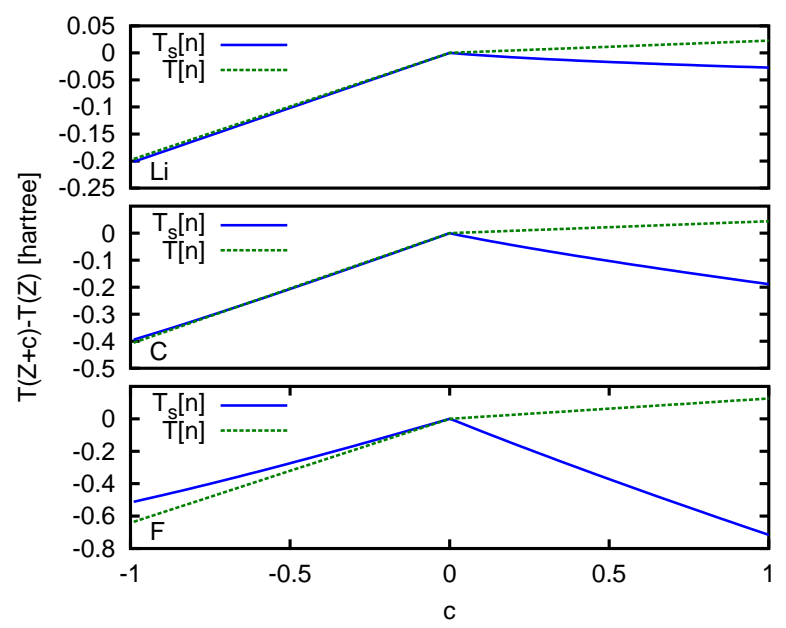

FIG. 4. (Color online) Change in Kohn-Sham kinetic energy $T_{s}^{Z+c}-T_{s}^{Z}$ and kinetic energy $T^{Z+c}-T^{Z}$ as a function of $c$.

follow an almost piecewise linear behaviour

$$
\varepsilon_{i}^{N} \approx(1-f)\left[\varepsilon_{i}^{M^{-}}+\Delta^{M}\right]+f \varepsilon_{i}^{(M+1)^{-}},
$$

where $i \leq N_{\text {inner. }}$ As far as we know, this is the first time that the energies of inner orbitals are studied as a function of the fractional electron number. We also plot in Figure 4 the KS kinetic energy $T_{s}^{Z+c}$ and the exact kinetic energy $T^{Z+c}=-E^{Z+c}$ (by the virial theorem) as a function of $c$, adjusted by their neutral atomic $c=0$ values. Unlike $T, T_{s}$ is not piecewise linear but was theoretically shown to be a convex function of $f[36]$. Our numerical results confirm this convex behavior. However, for all the systems studied here, $T_{s}$ turns out to be remarkably close to linearity

$$
T_{s}^{N} \approx(1-f) T_{s}^{M}+f T_{s}^{M+1},
$$

with the maximum deviation from linearity being -3.3 mhartree for $\mathrm{Li}^{-0.4},-8.9$ mhartree for $\mathrm{C}^{-0.4}$ and -15.3 mhartree for $\mathrm{F}^{+0.6}$. This near linearity was previously observed only for weakly interacting two-electron systems [37].

The near linearity of $T_{s}^{N}$ and $\varepsilon_{i}^{N}$ suggest that the KS orbitals change in a predictable fashion as partial electrons are added to the atoms, even in the difficult, degenerate systems considered here. If Eqs. (13) and (14) apply more generally they could provide desirable local and global constraints on new DFAs. Furthermore, since the KS kinetic energy $T_{s}^{N}$, the KS potential $v_{\mathrm{s}}^{N}(\boldsymbol{r})$, and the orbital energies $\varepsilon_{i}^{N}$ are linked by the KS energy expression $T_{s}^{N}+\int n^{N}(\boldsymbol{r}) v_{\mathrm{s}}^{N}(\boldsymbol{r}) \mathrm{d} \boldsymbol{r}=\sum_{i} f_{i} \varepsilon_{i}^{N}$ at fractional electron number $N$, the linear approximations of Eqs. (13) and (14) suggest a linear approximation for $n^{N}(\boldsymbol{r}) v_{\mathrm{s}}^{N}(\boldsymbol{r})$

$$
\begin{aligned}
n^{N}(\boldsymbol{r}) v_{\mathrm{s}}^{N}(\boldsymbol{r}) \approx & (1-f) n^{M}(\boldsymbol{r})\left[v_{\mathrm{s}}^{M^{-}}(\boldsymbol{r})+\Delta^{M}\right] \\
& +f n^{M+1}(\boldsymbol{r}) v_{\mathrm{s}}^{(M+1)^{-}}(\boldsymbol{r}) .
\end{aligned}
$$

Indeed, we found that Eq. (15) is an excellent approximation to $v_{\mathrm{s}}^{N}(\boldsymbol{r})$ for all cases studied here, especially when considered against the electronic density. Details are provided in the supplementary material [30]. We note that this approximation becomes exact in two limits: close to a nucleus where the KS potentials are dominated by the diverging electron-nucleus potential $v_{\text {ext }}(\boldsymbol{r})$, and in the asymptotic region $|\boldsymbol{r}| \rightarrow \infty$ where the density $n^{M+1}(\boldsymbol{r})$ dominates over $n^{M}(\boldsymbol{r})$ and the KS potential $v_{\mathrm{s}}^{N}(\boldsymbol{r})$ reduces to $v_{\mathrm{s}}^{(M+1)^{-}}(\boldsymbol{r})$. It can also be shown that this approximation is exact in the trivial case $0 \leq N \leq 1$ and in the case $1 \leq N \leq 2$ if $n^{2}(\boldsymbol{r})=2 n^{1}(\boldsymbol{r})$. Preliminary work suggests that this approximation may be valid in a wider range of systems than those explored here.

Conclusions. We have shown that accurate KS potentials $v_{\mathrm{s}}^{N}(\boldsymbol{r})$ at non-integer electron numbers $N$ can be obtained by inversion of accurate $a b$ initio electron densities. This has allowed us to numerically reproduce on systems with more than two electrons the theoretically predicted spatially constant discontinuity of the KS potential when the electron number crosses an integer. We have also found that, for all the atomic systems studied here, both the energies $\varepsilon_{i}^{N}$ of the inner orbitals (below the HOMO) and the KS kinetic energy $T_{s}^{N}$ are nearly piecewise linear functions of $N$. This has lead us to propose the simple approximation of Eq. (15) for the KS potential $v_{\mathrm{s}}^{N}(\boldsymbol{r})$ at any fractional electron number $N$ which uses only quantities of the systems with adjacent integer electron numbers. This approximation appears to work very well for all the cases considered and its generality and potential application to fragment and partition DFT [38-40] will be explored in future work.

\section{ACKNOWLEDGMENTS}

TG received computing support from the Griffith University Gowonda HPC Cluster. 
[1] W. Kohn and L. J. Sham, Phys. Rev. 140, A1133 (1965).

[2] P. Hohenberg and W. Kohn, Phys. Rev. 136, B864 (1964).

[3] J. P. Perdew, K. Burke, and M. Ernzerhof, Phys. Rev. Lett. 77, 3865 (1996).

[4] A. D. Becke, J. Chem. Phys. 98, 1372 (1993).

[5] J. P. Perdew, in Density Functional Methods In Physics, NATO ASI Series, Vol. 123, edited by R. M. Dreizler and J. da Providência (Springer US, 1985) pp. 265-308.

[6] A. J. Cohen, P. Mori-Sánchez, and W. Yang, Chem. Rev. 112, 289 (2012)

[7] J. P. Perdew, R. G. Parr, M. Levy, and J. L. Balduz, Phys. Rev. Lett. 49, 1691 (1982).

[8] At least under certain assumptions about orbital continuity.

[9] J. P. Perdew and M. Levy, Phys. Rev. Lett. 51, 1884 (1983).

[10] L. J. Sham and M. Schlüter, Phys. Rev. Lett. 51, 1888 (1983).

[11] N. T. Maitra, J. Chem. Phys. 122, 234104 (2005).

[12] A. Ruzsinszky, J. P. Perdew, G. I. Csonka, O. A. Vydrov, and G. E. Scuseria, J. Chem. Phys. 125, 194112 (2006).

[13] P. Mori-Sánchez, A. J. Cohen, and W. Yang, J. Chem. Phys. 125, 201102 (2006).

[14] A. Ruzsinszky, J. P. Perdew, G. I. Csonka, O. A. Vydrov, and G. E. Scuseria, J. Chem. Phys. 126, 104102 (2007).

[15] A. J. Cohen, P. Mori-Sánchez, and W. Yang, Science 321, 792 (2008).

[16] P. Mori-Sánchez, A. J. Cohen, and W. Yang, Phys. Rev. Lett. 100, 146401 (2008).

[17] O. A. Vydrov, G. E. Scuseria, and J. P. Perdew, J. Chem. Phys. 126, 154109 (2007).

[18] A. J. Cohen, P. Mori-Sánchez, and W. Yang, J. Chem. Phys. 126, 191109 (2007).

[19] P. Mori-Sánchez, A. J. Cohen, and W. Yang, Phys. Rev. Lett. 102, 066403 (2009).

[20] E. R. Johnson and J. Contreras-García, J. Chem. Phys. 135, 081103 (2011).
[21] T. Gould and J. F. Dobson, J. Chem. Phys. 138, 014103 (2013).

[22] E. Kraisler and L. Kronik, Phys. Rev. Lett. 110, 126403 (2013).

[23] G. K.-L. Chan, J. Chem. Phys. 110, 4710 (1999).

[24] W. Yang, Y. Zhang, and P. W. Ayers, Phys. Rev. Lett. 84, 5172 (2000).

[25] A. Savin, Chem. Phys. 356, 91 (2009).

[26] O. V. Gritsenko and E. J. Baerends, J. Chem. Phys. 120, 8364 (2004).

[27] K. Boguslawski, C. R. Jacob, and M. Reiher, J. Chem. Phys. 138, 044111 (2013).

[28] J. Toulouse, R. Assaraf, and C. J. Umrigar, J. Chem. Phys. 126, 244112 (2007).

[29] J. Toulouse and C. J. Umrigar, J. Chem. Phys. 128, 174101 (2008).

[30] See supplementary material.

[31] Y. Wang and R. G. Parr, Phys. Rev. A 47, R1591 (1993).

[32] P. Gori-Giorgi and A. Savin, Int. J. Quantum Chem. 109, 2410 (2009).

[33] J. P. Perdew and E. Sagvolden, Can. J. Chem. 87, 1268 (2009).

[34] E. Sagvolden and J. P. Perdew, Phys. Rev. A 77, 012517 (2008).

[35] The asymptotic behavior of the potential is determined by the asymptotic behavior of the ensemble density in Eq. (4) which is dominated for large $r$ by the $(M+1)$ electron density.

[36] M. Levy, J. S. M. Anderson, F. H. Zadeh, and P. W. Ayers, J. Chem. Phys. 140, (2014).

[37] P. Gori-Giorgi and A. Savin, J. Phys.: Conf. Ser. 117, 012017 (2008).

[38] P. Elliott, K. Burke, M. H. Cohen, and A. Wasserman, Phys. Rev. A 82, 024501 (2010).

[39] R. Tang, J. Nafziger, and A. Wasserman, Phys. Chem. Chem. Phys. 14, 7780 (2012).

[40] E. Fabiano, S. Laricchia, and F. D. Sala, J. Chem. Phys. 140, (2014). 